\title{
6 Methodik
}

Die in den vorangehenden Kapiteln aufgestellten Hypothesen werden anhand von Daten aus einer im Jahr 2015 durchgeführten quantitativen Umfrage sowie einer Dokumentenanalyse an den Hochschulen Ludwigshafen und Worms geprüft. Dieses Kapitel stellt zunächst die Entwicklung des Erhebungsinstruments der quantitativen Erhebung dar, betrachtet die Durchführung der Erhebung sowie die Datenqualität und gibt einen Überblick über die Stichprobe. Im Anschluss werden die Auswertungsmethoden näher dargestellt. Das Kapitel schließt mit einem Überblick über die Dokumentenanalyse.

\subsection{Auswahl der Erhebungsinstrumente}

Das Erhebungsinstrument für die quantitative Befragung wurde aufgrund der Fragestellung sowie des Forschungsstands gewählt. Bezogen auf den Forschungsstand ist das Feld der Studierbarkeit - auch wenn nicht explizit der strukturellen Studierbarkeit - durch zahlreiche Evaluationen im Rahmen der Qualitätssicherung an Hochschulen exploriert (z. B. Krempkow und Bischof 2010; Pohlenz 2008; Steinhardt 2011b). Zu mehreren in dieser Arbeit genutzten Themen wurden bereits Skalen entwickelt, die entweder übernommen oder angepasst werden können. Zur strukturellen Studierbarkeit, also bei Elementen der Studienstruktur wie E-Learning oder der zeitlichen Gestaltung von Studiengängen, liegt bisher wenig quantitative oder qualitative Forschung vor. In diesem Fall ist die Entwicklung von neuen Skalen notwendig.

Diese Arbeit zielt darauf ab, das theoretisch entwickelte Konzept struktureller Studierbarkeit zu überprüfen und die Präferenzen von unterschiedlichen Studierendengruppen zu Studienstrukturen $\mathrm{zu}$ ermitteln. Um repräsentative Aussagen $\mathrm{zu}$ den genannten Fragestellungen treffen $\mathrm{zu}$ können, ist die Grundgesamtheit bestmöglich abzubilden. Im Befragungsdesign wird daher eine Vollerhebung angestrebt. Um das Konzept struktureller Studierbarkeit prüfen zu können, ist Varianz auf Ebene der Studienstrukturen notwendig (z. B. Anzahl der SWS oder Wahlmöglichkeiten). Daher werden alle unterschiedlichen Fachrichtungen sowie zwei Hochschulen in die zu befragende Grundgesamtheit aufgenommen. Die Varianz in den Rahmenbedingungen, also bezogen auf den Hochschul- oder Studiengangtyp und die gesetzlichen Regelungen, wird hingegen möglichst klein gehalten. Um diese ähnlichen Bedingungen zu ermöglichen, wurden Studierende grundständiger Bachelor- und konsekutiver Masterstudiengänge an zwei Fachhochschulen in Rheinland-Pfalz befragt. Duale 
Studiengänge oder Weiterbildungsstudiengänge wurden von der Befragung ausgeschlossen, da die Studienstrukturen und die Lebenssituationen der Studierenden dieser Studiengänge sich deutlich von denen der Studierenden grundständiger oder konsekutiver Vollzeitstudiengänge unterscheiden. Darüber hinaus richtete sich die Befragung an Studierende im mindestens zweiten Bachelorsemester. Hierdurch sollte sichergestellt werden, dass die Studierenden die Studiensituation anhand eigener Erfahrungen einschätzen können. Als Methodik, die sich für eine große Anzahl an Befragten eignet, kam die quantitative Befragung zum Einsatz.

Bei der quantitativen Befragung von Studierenden werden deren Einschätzungen und Präferenzen zu Studienstrukturen erfragt. Will man objektive Aussagen zur aktuellen Studienstruktur erhalten, empfiehlt sich hingegen eher eine Dokumentenanalyse. Hierbei werden die Prüfungsordnungen, Modulhandbücher und Stundenpläne von Studiengängen anhand eines Analyseschemas bezogen auf ihre Ermöglichung von Flexibilität kategorisiert. In der Phase der Datenanalyse werden diese Daten dem Datensatz hinzugefügt. Eine nähere Beschreibung der Dokumentenanalyse findet sich in Kapitel 6.5.

\subsection{Entwicklung des Fragebogens}

Die Entwicklung des Fragebogens für die quantitative Befragung erfolgte federführend durch die Autorin dieser Dissertation in Zusammenarbeit mit dem Team des Projektes „Offenes Studienmodell Ludwigshafen“, da die Ergebnisse auch für die hochschulinterne Organisationsentwicklung genutzt wurden $(\mathrm{Bu} ß$ et al. 2016). Die Entwicklung der Fragen und der Merkmalsausprägungen erfolgte einerseits durch die Adaption bestehender Fragenbatterien und andererseits durch die konzeptgeleitete Entwicklung neuer Fragen unter Hinzuziehung von Interviews mit Studierenden und einer Literatur- bzw. Fragebogenrecherche (Kallus 2010, 24 ff.). Im Folgenden werden zunächst die Kriterien für die Fragebogenentwicklung hergeleitet, außerdem wird ein Überblick über die Items und Skalen des Fragebogens gegeben (der Fragebogen findet sich in Anhang 2). Im Anschluss folgt eine Zusammenfassung des Pretests.

\subsubsection{Kriterien bei der Fragebogenentwicklung}

Bei der Entwicklung und Testung von Fragebögen sind mehrere Gütekriterien zu berücksichtigen. Erstens definiert die Objektivität den „Grad, in dem das Untersuchungsresultat unabhängig ist von jeglichen Einflüssen außerhalb der untersuchten Person“ (Rost 1996, S. 39). Dabei beinhaltet die Objektivität mehrere 
Aspekte, so gleichbleibende Untersuchungsbedingungen (Durchführungsobjektivität), Einheitlichkeit der Auswertung von Messwerten (beispielsweise bei offenen Fragen, Auswertungsobjektivität) und Vergleichbarkeit der Ergebnisinterpretation unterschiedlicher Forscher (Interpretationsobjektivität) (Bühner 2006, S. 36 ff.; Rammstedt 2010, S. 241 ff.). Zweitens prüft die Reliabilität, wie genau eine „Skala ein Merkmal misst“" (Rammstedt 2010, S. 242). Dies kann beispielsweise durch die Wiederholung einer Befragung oder die Berücksichtigung mehrerer Skalen, die das gleiche Konzept erfassen, geschehen. Die Validität bestimmt drittens, inwiefern eine Befragung das misst, was sie messen soll. Die Inhaltsvalidität prüft dabei, ob die Items eines Fragebogens alle relevanten Elemente eines Merkmalsbereichs beinhalten. Die Kriteriumsvalidität nimmt in den Blick, ob die Ergebnisse der Befragung mit weiteren Erkenntnissen zu dem Thema (z. B. objektiven Studienabbruchzahlen, Noten) zusammenhängen. Die Konstruktvalidität prüft schließlich, ob das Messinstrument das zu erfassende Konstrukt tatsächlich erfasst (Rammstedt 2010, S. 249 ff.). In diesem Kapitel sowie in Unterkapitel 6.3 steht die Frage im Mittelpunkt, wie diese Gütekriterien bei der Fragebogenentwicklung und Testung berücksichtigt wurden.

Um eine hohe Validität zu erreichen und das Konzept struktureller Studierbarkeit korrekt abzubilden, wurde besonderes Augenmerk auf die inhaltliche Erfassung der Items und passende Antwortformate gelegt. Dabei stehen logisch-fachliche Überlegungen im Mittelpunkt - es sind insbesondere die Formulierung aus Sicht der Befragten, die Eindeutigkeit der Konzepte und die Relevanz für alle Befragten zu beachten (Bühner 2006, S. 36; Kallus 2010, S. 17, 55 ff.). Um eine hohe Inhaltsvalidität zu erreichen, dienten die bisherige Forschung (siehe Kapitel 5) und qualitative Interviews als Grundlage der ItemFormulierung. Im Rahmen von Einzelinterviews mit sechs Studierenden ${ }^{10}$ (Zusammenfassung bei Müller et al. 2015, S. 14 ff.) und eines darauffolgenden Gruppengesprächs mit den gleichen Studierenden wurde thematisiert, welche Studienstrukturen das Studierverhalten der befragten berufstätigen Studierenden und Studierenden mit Kind besonders beeinflussen. Die interviewten Studierenden nannten passende Lehrveranstaltungszeiten und das Angebot von Blockveranstaltungen, ein hohes $\mathrm{Ma}$ an Wahlmöglichkeiten, E-Learning, ein Teilzeitstudium und die Flexibilität der Fristen besonders häufig. Diese Aspekte, die sich mit den aus der Literatur entwickelten Elementen struktureller Studierbarkeit decken (siehe Kapitel 2.1), wurden im darauffolgenden Gruppengespräch konkretisiert. Es wurde deutlich, dass die Studierenden unterschiedlicher Studiengänge einzelne Studienstrukturen unterschiedlich definieren. Die Definition hängt von den eigenen Erfahrungen im Studiengang

${ }^{10}$ Durchgeführt durch Barbara Husemann, wissenschaftliche Mitarbeiterin im Projekt Offenes Studienmodell Ludwigshafen. 
sowie der subjektiven Bewertung dieser Erfahrungen ab. Diese Aspekte wurden insofern berücksichtigt, als die Items die Konzepte mit den Worten der Studierenden erklären und negative Erfahrungen durch die Beschreibung von Qualitätsanforderungen reduzieren. Beispielsweise verstehen Studierende unter Blockveranstaltungen ganz unterschiedliche Praktiken, sodass gemeinsam mit den Studierenden die Formulierung ,ganztägig geblockte Veranstaltungen, die an zwei bis drei Terminen stattfinden" erarbeitet wurde. Ein anderes Beispiel sind die negativen Erfahrungen mit E-Learning und eine große Bedeutungsbandbreite des Begriffs E-Learning. Daher wurde die Frage dahingehend konkretisiert, dass es sich bei E-Learning um den Ersatz von Präsenzveranstaltungen handelt und die Lehrenden die virtuellen Phasen aktiv begleiten.

Die Inhaltsvalidität ist auch bei der Bemessung der in das Studium investierten Zeit, also beim Workload, äußerst relevant. Im Gegensatz zu vielen Zeitlasterhebungen (z. B. Schulmeister und Metzger 2011, S. 22) wird in dieser Arbeit nicht die tatsächliche Lernzeit und Präsenzzeit gemessen. Hierfür liegen zwei Gründe vor: Erstens ist die retrospektive Einschätzung der Lernzeiten durch Studierende wenig valide, da Studierende oft große Schwierigkeiten bei der korrekten Einschätzung ihrer auf das Studium verwendeten Zeit pro Tag oder Woche haben (Stinebrickner und Stinebrickner 2004, S. 244 f.). Die Nutzung von Tagebüchern zur Dokumentation der Lernzeiten hätte einen zu großen Aufwand bedeutet und wäre gleichzeitig bei einer erwünschten Fallzahl von mindestens 1.000 Studierenden kaum realisierbar gewesen. Zweitens ist die Gesamtheit der Studierenden sehr heterogen, und nicht alle sind auf die gleiche Anwesenheitsquote bei Veranstaltungen oder die gleiche Selbstlernzeit angewiesen. Daher wird im Fragebogen die subjektive Einschätzung gemessen, ob die Studierenden Schwierigkeiten haben, Veranstaltungen und Prüfungen zu besuchen oder Selbstlernzeiten einzurichten.

Neben der verständlichen Formulierung der Items sind für die Kriteriumsund Konstruktvalidität und die Reliabilität Vergleiche mit vor oder nach dem Test erhobenen Daten, der Vergleich mit ähnlichen Befragungen oder die Wiederholung der Befragung sinnvoll (Bühner 2006, S. 38 ff.; Rammstedt 2010, S. 244). Diese Prüfungen sind nur eingeschränkt möglich, da bisher keine anderen Befragungen zu struktureller Studierbarkeit vorliegen. Auch der Abgleich mit zeitlich nachgelagerten Daten - dazu eignen sich beispielsweise Abbruchquoten oder Studiendauern - ist nicht umsetzbar, denn diese Daten dürfen aus Datenschutzgründen derzeit nicht mit den Befragungsdaten verbunden werden. Als Vergleich mit ähnlichen Befragungen können daher lediglich die von anderen Autorinnen und Autoren übernommenen Skalen (z. B. Studienzufriedenheit nach Westermann) anhand der Teststatistiken dienen. 
Um Verzerrungen (Bühner 2006, S. 68 ff.) zu vermeiden, wurden folgende Maßnahmen vorgenommen: Die Formulierungen der Items sind so gewählt, dass sie eindeutig zu beantworten sind, nur einen Aspekt pro Aussage beinhalten und die Aussagen für alle Befragten ausreichend häufig vorkommen. Private oder sehr komplexe Fragen bleiben häufiger unbeantwortet als unpersönliche und einfache Items. Aus diesem Grund sind die Fragen inhaltlich und grammatikalisch einfach gehalten und beinhalten in der Regel einen Skalenpunkt „weiß ich nicht“ oder „keine Angabe“. Um zu häufiges Umdenken in den Antwortmöglichkeiten zu vermeiden, kommen neben Nominalskalen überwiegend Likertskalen (,trifft voll zu“ bis „trifft gar nicht zu“) zur Anwendung. An den Stellen, an denen diese Skala nicht passt, kommen Intensitätsskalen (,sehr häufig“ bis „gar nicht“) oder merkmalsbezogene Skalierungen (z. B. „,zu hoch“, „,zu niedrig“) zur Anwendung (Kallus 2010, S. 44 ff.). Alle Skalen sind als 5er-Skalen ausgestaltet und mit einer Ausnahme endpunktbenannt (Gerich 2010, S. 275). Die Ausnahme bildet die Frage nach der Zufriedenheit mit Studienstrukturen, bei der die fünf Skalenpunkte von „zu gering“ über „genau richtig“ bis hin zu „zu hoch“ spezifiziert sind und damit ordinalskaliert sind.

Verzerrungen durch soziale Erwünschtheit waren aufgrund der Fragestellung nicht $\mathrm{zu}$ erwarten. Eine Ausnahme bildet das Studierverhalten, bei dem die Abwesenheit bei Lehrveranstaltungen und der Umfang der Selbstlernzeit erfragt wurden. Diese Fragen hätten infolge sozialer Erwünschtheit zu positiv beantwortet werden können, da regelmäßiges Lernen und die Anwesenheit bei Lehrveranstaltungen als gutes Studierverhalten gilt. Um diesem Problem entgegenzuwirken, beinhaltet das Item durch die Einbettung in den Kontext der Vereinbarkeit einen klaren situativen Bezug und wurde wertneutral formuliert (Kallus 2010, S. 54; Sedlmeier und Renkewitz 2013, S. 93 f.). Bei der Gestaltung des Fragebogens wurde darauf geachtet, die Verzerrungen durch die Fragenabfolge möglichst gering zu halten. Um das Interesse der Studierenden zu wecken, beginnt der Fragebogen mit der Abfrage der Studien- und Lebenssituation und der studienstrukturellen Präferenzen der Studierenden. Persönliche Fragen zur Integration in die Hochschule, Überschreitung der Studiendauer oder der Behinderung hingegen sind im hinteren Teil des Bogens platziert. Ein offenes Feld zum Schluss des Fragebogens ermöglichte es den Studierenden, weitere Hinweise zum Thema der Befragung zu geben.

Die Befragung ist als Querschnitt angelegt. Um Ursache-WirkungsZusammenhänge und damit die Kausalrichtung belegen zu können, ist jedoch eine zeitliche Präzedenz zu berücksichtigen (Sedlmeier und Renkewitz 2013, S. 124 ff.). Die Ursache muss zeitlich vor der Wirkung liegen und gemessen werden. In der vorliegenden Befragung konnten daher statt Kausalitäten lediglich ungerichtete Zusammenhänge bzw. Korrelationen erfasst werden. Das Modell der 
strukturellen Studierbarkeit wird in dieser Arbeit über einzelne Regressionen getestet. Eine grafische Darstellung aller geprüften Zusammenhänge des Modells findet sich in Kapitel 7.4.

Tabelle 13 gibt eine Übersicht über die neun Bereiche des Fragebogens und ihre Anordnung, die zugrunde liegende Literatur der Fragen sowie die Skalen und Skalenniveaus. 


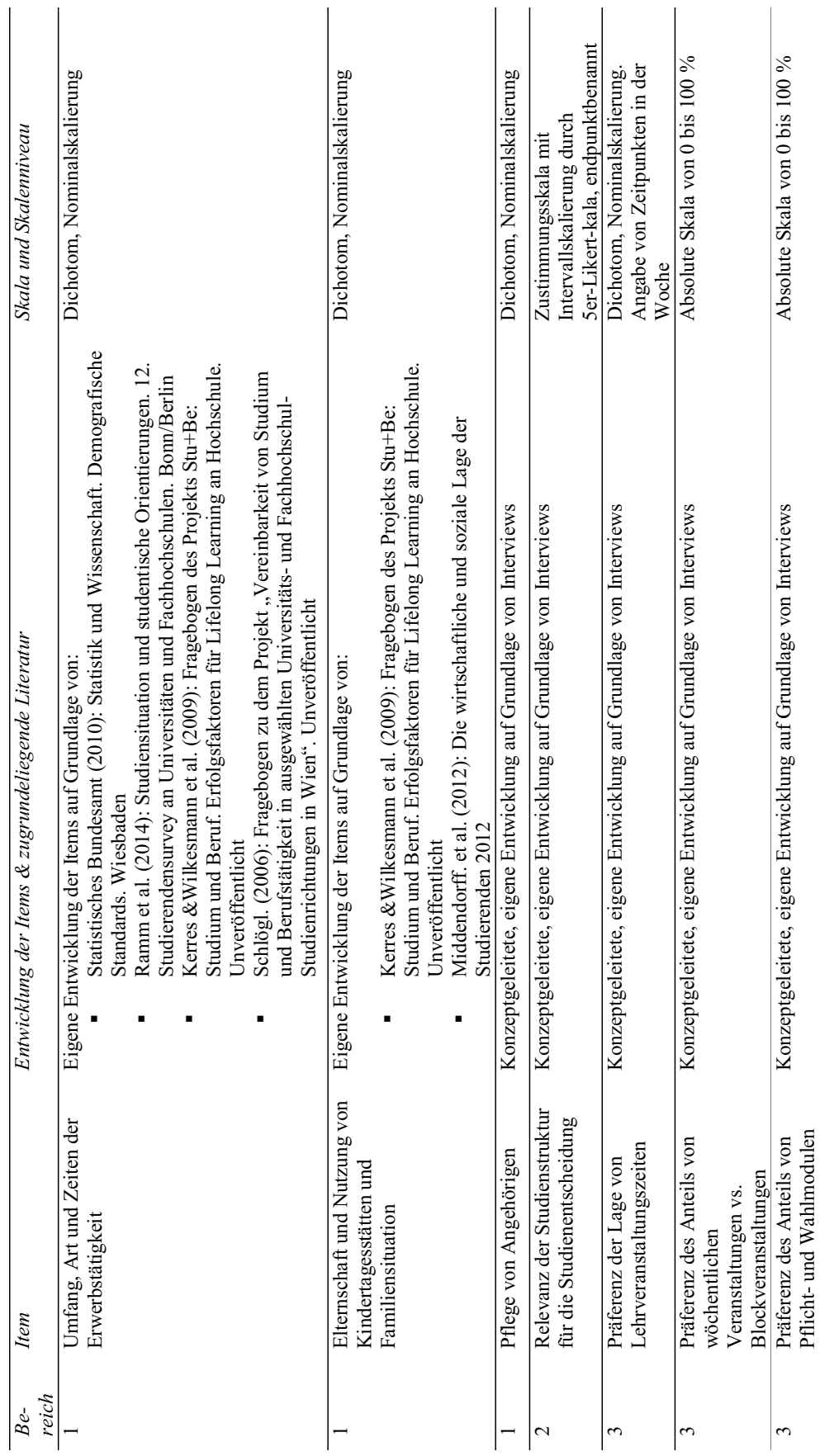




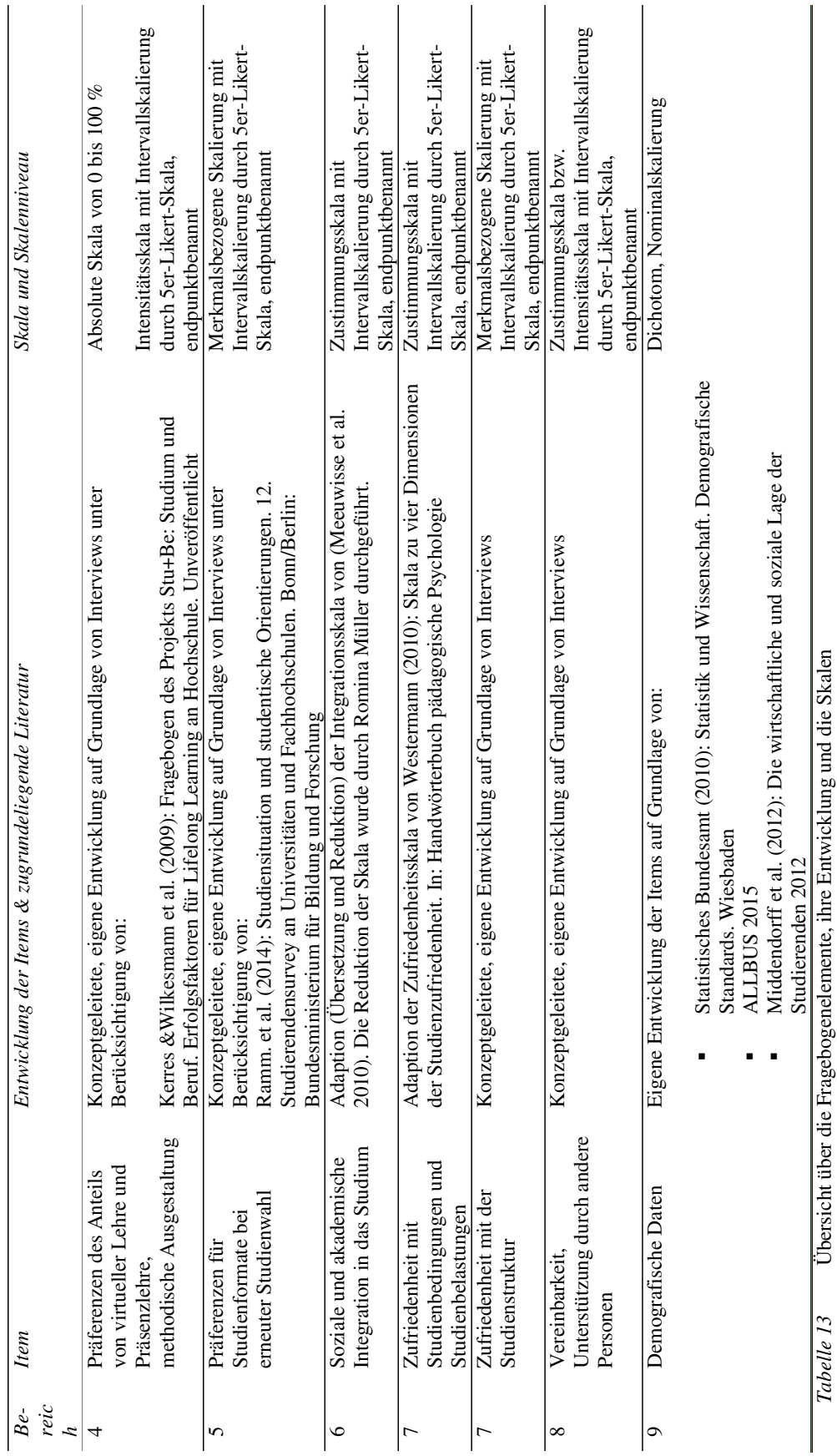




\subsubsection{Fragebogentestung}

Nach der Ersterstellung des Fragebogens erfolgte ein Pretest mit 50 Studierenden aus zwei Disziplinen der Hochschule Ludwigshafen. Alle teilnehmenden Studierenden füllten den Test im Rahmen einer Lehrveranstaltung aus, die Lehrenden stellten hierfür eine halbe Stunde Zeit zur Verfügung. Aus den Ergebnissen wurde eine Teststatistik zur Prüfung der Reliabilität (Mittelwerte, Streuung, fehlende Werte, Itemschwierigkeit, Trennschärfe, Cronbachs Alpha) bzw. Validität (Faktorenanalyse) erstellt (Rammstedt 2010, S. 256). Auf Grundlage dieser Tests wurden die Skalen zur Integration und Vereinbarkeit angepasst und insbesondere die Skalen in ihrer Anzahl leicht reduziert. Mehrere Fragen wiesen eine große Anzahl fehlender Werte auf. Genau diese Fragen thematisierten auch die acht Studierenden im kognitiven Pretest, welcher zusätzlich zur Testbefragung durchgeführt wurde. Bei dem kognitiven Pretest zeigte sich, dass nur Hälfte bis zwei Drittel der Teilnehmer die Konzepte ELearning, Blockveranstaltungen und Wahlmodule wie intendiert verstanden. Daher wurden die Fragen konkretisiert und die Antwortmöglichkeiten „weiß ich nicht" oder ,keine Angabe“ systematisch in den Fragebogen aufgenommen. Alle anderen Items im Fragebogen wurden als gut verständlich eingeschätzt, darüber hinaus wurde der gute Aufbau des Fragebogens gelobt.

\subsection{Durchführung der Erhebung}

Die Durchführung der Befragung wird im Folgenden anhand des Erhebungsablaufs und der Prüfung der Datenqualität dargelegt.

\subsubsection{Ablauf und Art der Erhebung}

Um eine hohe Übertragbarkeit und Objektivität der Daten zu gewährleisten, war ein hoher Rücklauf im Sinne einer Vollerhebung Ziel der Durchführungskonzeption. An der Hochschule Ludwigshafen konnte dies erreicht werden. Die Befragung fand in Ludwigshafen als Paper-Pencil-Befragung in einem Zeitraum von zwei Wochen in den am besten besuchten Präsenzveranstaltungen jedes Semesters statt. Um eine hohe Abdeckung zu erzielen und die Wahrscheinlichkeit der Teilnahme aller Gruppen zu erhöhen, fand die Befragung in der Mitte des Semesters statt. Zu diesem Zeitpunkt befinden sich erfahrungsgemäß die meisten Studierenden am Hochschulstandort. Die Lehrenden der ausgewählten Veranstaltungen räumten dem Projektteam etwa 20 Minuten 
ihrer Veranstaltungszeit ein, um die Befragung durchzuführen. Die Akzeptanz der Studierenden war sehr hoch, sodass mit sehr wenigen Ausnahmen alle Anwesenden den Fragebogen ausfüllten.

Durch eine Kooperation mit der Hochschule Worms konnte die Befragung auch dort durchgeführt werden. Da die personellen Ressourcen keine PaperPencil-Befragung zuließen, wurde die Umfrage als Onlinebefragung durchgeführt. Der Fragebogen wurde mit zwei Erinnerungen per Mail an die Studierenden der Hochschule versendet. Hierdurch hatten auch Studierende des ersten Semesters die Möglichkeit, zu antworten, welche in Ludwigshafen nicht angesprochen worden waren. Diese Antworten wurden in den Analysen ausgeschlossen. Wegen der Form der Onlinebefragung war die Beteiligung an der Hochschule Worms deutlich geringer als in Ludwigshafen (siehe dazu Kapitel 6.3.2).

Neben der Form der Erhebung wurde auf gleiche Bedingungen bei der Information zur Befragung geachtet, um eine hohe Durchführungsobjektivität zu erreichen (Rammstedt 2010, S. 240). Eine Mitarbeiterin aus dem Projektteam „Offenes Studienmodell Ludwigshafen“ informierte die Studierenden über die Ziele der Befragung, die Freiwilligkeit und die Datenschutzrichtlinien sowie die Verwendung der Ergebnisse. An der Hochschule Worms wurden diese Informationen stattdessen in einer Mail sowie auf der Homepage der Befragung zur Verfügung gestellt. Um auch eine persönliche Ansprache zu ermöglichen, konnten Studierende aus Worms den Telefon- oder Mailkontakt zu Ansprechpartnerinnen suchen.

Die Daten wurden nach der Erhebung via EvaSys eingelesen. Lediglich die Fragen mit Prozentangaben sowie die Bezeichnungen der Art der Erwerbstätigkeit mussten nachträglich händisch digitalisiert werden, sodass die Auswertungsobjektivität als hoch einzuschätzen ist (Rammstedt 2010, S. 242).

\subsubsection{Datenqualität}

Die Qualität der Daten und die Übertragbarkeit auf die Population der Studierenden an Fachhochschulen in ähnlichen Fächern ergibt sich aus dem Rücklauf und der Ähnlichkeit zwischen Stichprobe und Grundgesamtheit (Sedlmeier und Renkewitz 2013, 116 ff.). An der Hochschule Ludwigshafen nahmen 60 Prozent (980 Personen) der Grundgesamtheit teil - eine Quote, die als sehr gut zu bezeichnen ist. Bei der Berechnung der Grundgesamtheit wurden nur Studierende $a b$ dem zweiten Semester in grundständigen Bachelor- und konsekutiven Masterstudiengängen berücksichtigt, die sich zum Zeitpunkt der Befragung an der Hochschule befanden. Studierende in Auslands- oder Praxissemestern zählen nicht dazu. Verzerrungen können entstehen, wenn die Stichprobe die Grundgesamtheit nicht gut abbildet. In den Ludwigshafener Daten 
kann es zu kleinen Verzerrungen kommen, falls einige Studierendengruppen seltener an Lehrveranstaltungen teilgenommen haben. Die Vergleichsdaten aus der Studierendenstatistik in Tabelle 14 zum Anteil weiblicher Studierender und zum Studiengangtyp zeigen allerdings eine hohe Übereinstimmung zwischen der Stichprobe und der Grundgesamtheit mit Abweichungen von bis zu 5 Prozentpunkten bei dem Anteil weiblicher Studierender.

Tabelle $14 \quad$ Vergleich Rücklauf und Grundgesamtheit: Eigene Darstellung.

\begin{tabular}{lll} 
& HS Ludwigshafen & HS Worms \\
\hline Methodik & $\begin{array}{l}\text { Papierbefragung in } \\
\text { Lehrveranstaltungen }\end{array}$ & Onlinebefragung \\
\hline Rücklauf & $60 \%$ & $8 \%$ \\
\hline Anteil weiblich & & \\
Grundgesamtheit & $53 \%$ & $58 \%$ \\
Datensatz & $58 \%$ & $71 \%$ \\
\hline Anteil Bachelor & & \\
Grundgesamtheit & $88 \%$ & $80 \%$ \\
Datensatz & $85 \%$ & $80 \%$ \\
\hline
\end{tabular}

An der Hochschule Worms antworteten 272 Studierende. Die Berechnung der Grundgesamtheit ist in diesem Fall deutlich schwerer, da über die Onlinebefragung alle eingeschriebenen Studierenden angesprochen wurden - dies schließt Studierende im Auslands- oder Praxissemester ebenso ein wie Studierende in dualen Studiengängen in der Praxisphase. Der Rücklauf von 8 Prozent bezieht sich auf alle eingeschriebenen Studierenden in den grundständigen und konsekutiven Studiengängen und ist damit konservativ geschätzt. In Worms kann es zu Verzerrungen gekommen sein, da die Teilnahme an der Umfrage stark von der Motivation der Studierenden abhing. Es ist daher möglich, dass diese Stichprobe nicht vollständig zufällig entstanden ist. Indizien hierfür zeigt die Studierendenstatistik, da der Anteil der Studentinnen im Datensatz um 13 Prozentpunkte höher liegt, als in der Grundgesamtheit. Der Anteil der Bachelorstudierenden hingegen ist in der Grundgesamtheit und der Stichprobe identisch. Um weitere studentische Merkmale zu prüfen, werden im Folgenden die im Fokus stehenden Gruppen der Erwerbstätigen, der Studierenden mit Kind oder Pflegeaufgaben und der Studierenden mit Beeinträchtigung getrennt nach Hochschulen dargestellt. Da zu den genannten Aspekten keine gesicherten Daten der Grundgesamtheit vorliegen, ist eine Aussage $\mathrm{zu}$ den tatsächlichen Verzerrungen nicht möglich. Aus dem gleichen Grund können mögliche, aber nicht nachgewiesene Verzerrungen nicht durch eine Gewichtung der Stichprobe ausgeglichen werden. 
Die Datenqualität wurde durch verschiedene Maßnahmen in der Datenaufbereitung sichergestellt. Für alle Items wurden die Itemschwierigkeit und die Trennschärfe berechnet. Diese Werte waren akzeptabel, lediglich die Itemschwierigkeit war für die Frage „Schwierigkeit, an Prüfungen teilzunehmen“ und die damit zusammenhängende Frage zur Abbruchneigung niedrig. Trotz der geringen Werte werden die Items nicht ausgeschlossen, da das Antwortverhalten mit den geringen Abbruchquoten an den beiden Hochschulen übereinstimmt. Fehlende Werte und Extremwerte wurden von den Analysen ausgeschlossen. Zur Prüfung der Skalen wurden Cronbachs Alpha bzw. konfirmatorische Faktorenanalysen durchgeführt; die Ergebnisse sind in Anhang 1 nachzulesen. Sie zeigen, dass die nach dem Pretest erstellen Skalen eine gute interne Konsistenz aufweisen. Alle statistischen Verfahren wurden auf Modellverstöße getestet (siehe Kapitel 6.6).

\subsection{Beschreibung des Samples}

\subsubsection{Geschlecht, Alter und Herkunft}

Von den Befragten sind 61,2 Prozent weiblich und 38,2 Prozent männlich, Weitere 0,6 Prozent gaben ein anderes Geschlecht an. Die Überrepräsentanz von Frauen ist insbesondere auf die Befragten der Hochschule Worms zurückzuführen, da hier 71,2 Prozent der Befragten Frauen waren. Die Befragten waren durchschnittlich 24,5 Jahre alt, wobei die Masterstudierenden mit 26,1 Jahren durchschnittlich zwei Jahre älter waren als ihre Kommilitonen in Bachelorstudiengängen.

Zur Herkunft der Studierenden liegen Daten zum Bildungshintergrund der Eltern und zum Migrationshintergrund vor. 21,8 Prozent der Studierenden sind entweder selber zugewandert oder haben mindestens einen Elternteil, der nicht in Deutschland geboren ist. In 33 Prozent der Elternhäuser hat mindestens ein Elternteil einen Hochschulabschluss erworben. Somit studieren 67 Prozent der Studierenden als Erste der engeren Familie.

\subsubsection{Bildungsweg und Studiengänge}

Die Bildungswege der Studierenden sind sehr divers und unterscheiden sich deutlich von denen der Universitätsstudierenden. Abbildung 10 stellt dar, welche Schul- und Berufsabschlüsse die Befragten auf ihrem Bildungsweg erworben haben. Auch wenn 60 Prozent der Studierenden mit Abitur an die Hochschule gekommen sind, ging der Weg vieler Studierender über die Hauptschule (7 
Prozent) und die Realschule (34 Prozent). 5,8 Prozent der Befragten sind durch eine mit einem Notendurchschnitt von 2,5 oder besser bestandene mittlere Reife und eine zweijährige Ausbildung, einen Meister oder einen Abschluss als Techniker beruflich qualifiziert. Mit 63 Prozent hat die Mehrheit der Studierenden eine Ausbildung absolviert und ist damit schon mit dem Berufsleben in Kontakt gekommen.

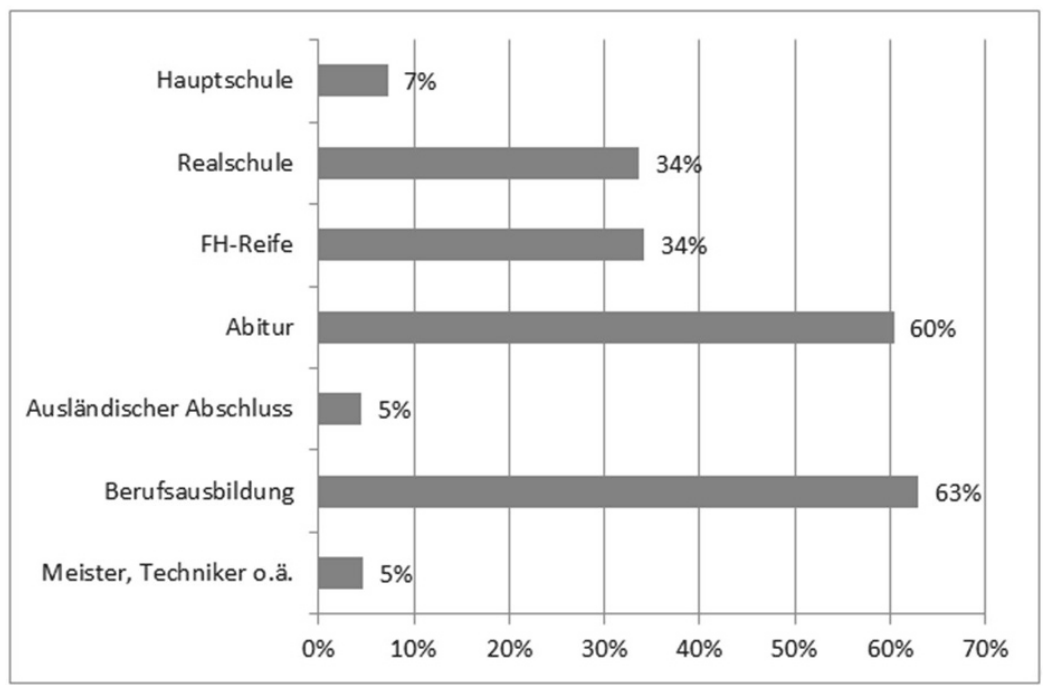

Abbildung 10 Schul- und Berufsabschlüsse der Befragten, Mehrfachantworten möglich.

Die Studierenden der befragten Hochschulen sind in 43 unterschiedlichen Studiengängen und mit 70,6 Prozent überwiegend im Bereich der Betriebswirtschaft eingeschrieben. Dabei sind die Studiengänge in den beiden Hochschulen als fachlich ausdifferenziert $\mathrm{zu}$ betrachten. Beispiele für Studiengänge mit hohen Studierendenzahlen sind Personalwesen, Marketing, Logistik, Handels- und Tourismusmanagement oder Controlling. Die mit 20 Prozent zweitgrößte Gruppe der Studierenden ist im Sozial- und Gesundheitswesen eingeschrieben und studiert Soziale Arbeit oder Pflegepädagogik. Die drittgrößte Gruppe sind Studierende der Fächer Informatik oder Wirtschaftsinformatik (9,4 Prozent). Insgesamt überwiegen Bachelorstudierende; lediglich 15,9 Prozent der Befragten sind in einem Masterstudiengang eingeschrieben. 


\subsubsection{Lebenssituation: Berufstätigkeit und Sorgeaufgaben}

Tabelle 15 stellt Umfang, Art und Bereich der Berufstätigkeit der befragten Studierenden dar. Die Lebenssituation der meisten Studierenden an den beiden Hochschulen ist von starker Erwerbstätigkeit geprägt, wobei die Studierenden an der Hochschule Ludwigshafen in höherem Umfang erwerbstätig sind. Insgesamt arbeitet die Hälfte der Studierenden weniger als 20 Stunden und damit in Teilzeit. Weitere 5,7 Prozent der Befragten arbeiten mehr als 20 Stunden pro Woche und damit in Vollzeit. Der Anteil der Erwerbstätigen liegt unter dem Bundesdurchschnitt an Fachhochschulen von 65 Prozent (Middendorff et al. 2017, S. 61). Interessant ist, dass ein Viertel der Erwerbstätigen in einem vorher erlernten Beruf tätig ist. Dies spiegelt sich auch in den Tätigkeiten wider, da fast ein Fünftel der Erwerbstätigen im Büro arbeitet und 14 Prozent im medizinischpflegerischen Bereich. 45 Prozent der erwerbstätigen Studierenden sind als Aushilfen beschäftigt und arbeiten eher in der Gastronomie, im Verkauf oder in sonstigen Tätigkeiten.

Die Sorgeaufgaben von Studierenden teilen sich in die Betreuung von Kindern und die Pflege von nahen Angehörigen auf. 4,7 Prozent der Studierenden haben Kinder, wobei der Anteil in Ludwigshafen deutlich höher liegt als in Worms. Dies geht besonders auf die in Ludwigshafen angebotenen Studiengänge im Sozial- und Gesundheitswesen zurück, da die Studierenden in diesen Fächern überproportional häufig Kinder haben. Im Vergleich mit dem Bundesschnitt von 5 Prozent an Fachhochschulen haben Studierende an den beiden untersuchten Hochschulen etwas seltener Kinder (Middendorff et al. 2017, S. 25). Ein Anteil von 4,6 Prozent der Befragten gab an, nahe Angehörige zu pflegen. Ein bundesweiter Vergleichswert aus der Sozialerhebung liegt nicht vor. Daten aus der bundesweiten CHE-Quest Befragung ergeben einen Anteil von 3,1 Prozent der befragten Studierenden, die Angehörige pflegen (Berthold und Leichsenring o. J.a, S. 20). Damit liegt der Wert in Ludwigshafen und Worms etwas höher als der Durchschnitt. 
Tabelle 15 Berufstätigkeit und Sorgeaufgaben der Befragten. Eigene Darstellung.

\begin{tabular}{|c|c|c|c|c|}
\hline Lebenssituation & $\begin{array}{l}\text { HS } \\
\text { Ludwigs- } \\
\text { hafen } \\
(\%)\end{array}$ & $\begin{array}{l}H S \\
\text { Worms } \\
(\%)\end{array}$ & $\begin{array}{l}\text { Gesamt } \\
(\%)\end{array}$ & $\begin{array}{l}\text { Bund } \\
(\%)\end{array}$ \\
\hline \multicolumn{5}{|l|}{ Erwerbsstätigkeit } \\
\hline Gesamt & 61,6 & 45,0 & 58,0 & 65,0 \\
\hline Teilzeit ( $<=20$ Stunden) & 53,8 & 37,5 & 50,2 & \\
\hline Vollzeit ( $>20$ Stunden) & 6,2 & 4,0 & 5,7 & \\
\hline \multicolumn{5}{|l|}{ Art Erwerbstätigkeit } \\
\hline Aushilfe & 47,8 & 34,2 & 45,5 & \\
\hline Stud./wiss. Hilfskraft & 3,5 & 6,7 & 4,1 & \\
\hline Werkstudent/in & 14,0 & 30,0 & 16,7 & \\
\hline Praktikum & 1,2 & 3,3 & 1,5 & \\
\hline Ausbildung (dual) & 0 & 8,3 & 1,4 & \\
\hline Voraussetzung: Ausbildung & 28,9 & 1,7 & 24,3 & \\
\hline Freiberuflich & 2,0 & 6,7 & 2,8 & \\
\hline Sonstiges & 2,0 & 5,0 & 2,5 & \\
\hline \multicolumn{5}{|l|}{ Arbeitsbereich } \\
\hline Service/Gastronomie & 6,2 & 11,7 & 7,2 & \\
\hline Bürotätigkeit & 14,5 & 44,2 & 19,5 & \\
\hline Verkauf & 4,2 & 15,8 & 6,2 & \\
\hline Medizinisch/pflegerisch & 17,4 & 0,8 & 14,6 & \\
\hline IT & 1,5 & 0,8 & 1,4 & \\
\hline Handwerklich & 3,0 & 1,7 & 2,8 & \\
\hline Pädagogisch/sozial & 8,3 & 14,2 & 9,3 & \\
\hline Architektur/Bau & 0,3 & 10,8 & 2,1 & \\
\hline Sonstiges & 44,4 & 0 & 36,9 & \\
\hline Kinder & 5,5 & 1,9 & 4,7 & 6,0 \\
\hline Davon mehr als 1 Kind & 2,8 & 0,7 & 2,3 & \\
\hline Pflegeaufgaben & 4,4 & 5,2 & 4,6 & 3,1 \\
\hline
\end{tabular}

\subsubsection{Beeinträchtigung und Behinderung}

An deutschen Hochschulen haben 23 Prozent der Studierenden eine gesundheitliche Beeinträchtigung. Dabei ist jedoch eine Differenzierung dieser Gruppe sinnvoll und notwendig, da sich anhand des sozialen Modells der 
Behinderung eine Behinderung aus zwei Aspekten zusammensetzt (siehe Unterkapitel 3.4). Die physische oder psychische Beeinträchtigung führt im Zusammenspiel mit gesellschaftlichen Verhältnissen zu Benachteiligungen und damit einer Behinderung (Hughes und Paterson 1997, S. 327). Diese gesellschaftlichen Verhältnisse können sich an Hochschulen so ausdrücken, dass Studierende durch ihre Beeinträchtigung im Studium behindert werden. Nur in diesem Fall werden sie als Studierende mit Behinderung bezeichnet. In Bundesschnitt haben 12 Prozent der Studierenden eine Beeinträchtigung und zusätzlich 11 Prozent eine Behinderung im Studium (Middendorff et al. 2017, S. 119). An den Hochschulen Ludwigshafen und Worms haben 3,1 Prozent der Studierenden eine Beeinträchtigung und zusätzlich 6,5 Prozent eine Behinderung. Diese Werte liegen deutlich unter dem Bundesschnitt, was unter anderem auf die Fächerzusammensetzung und den Hochschultyp zurückgeführt werden kann. Denn in den wirtschaftswissenschaftlichen Studiengängen geben auch laut der Sozialerhebung weniger Studierende eine Beeinträchtigung an (Middendorff et al. 2013, S. 459).

\subsection{Dokumentenanalyse}

Die Dokumentenanalyse in dieser Dissertation erfolgt anhand eines Schemas zur Analyse von studienstruktureller Studierbarkeit (Buß et al. 2015), wobei nur eine Auswahl der dort vorhandenen Variablen zur Anwendung kommt. Das gesamte Codierschema beinhaltet 21 verschiedene Variablen, welche die Struktur und Flexibilität im Studienalltag, die zeitliche Passung zu den Bedarfen der Zielgruppen, die Möglichkeiten bei Nichtteilnahme an Veranstaltungen sowie die Wertschätzung unterschiedlicher Bildungsbiografien beinhalten. Dabei sind immer mehrere Kategorien möglich, zum Beispiel bei der Berechnung der Semesterwochenstunden die Kategorie eins mit über 24 SWS, Kategorie zwei mit 21 bis 24 SWS und Kategorie drei mit 18 bis 20 SWS. Um die SWS-Anzahl zu ermitteln, wurden alle SWS im Studiengang durch die Anzahl der Semester geteilt, Semester mit hoher Abwesenheit durch Praktika oder die Abschlussarbeit ausgenommen. Die Festlegung der Kategorien erfolgte, wenn möglich, anhand vorliegender Forschungsarbeiten. Im Falle der Semesterwochenstunden hingegen entstammt die Einschätzung einer hohen Flexibilität bei 18 bis 20 SWS einer Diskussionsrunde mit Lehrenden und Studierenden. Das gesamte Codierschema ist bei $\mathrm{Bu}$ et al. (2015) nachzulesen.

Es wurden nur Strukturen in die Berechnungen aufgenommen, welche auf der Studiengangebene festgelegt werden und damit dort Varianz erzeugen. Gleichzeitig beschreiben die Variablen die zeitliche und örtliche Flexibilität von 
Studierenden, sodass fünf Variablen mit jeweils zwei Ausprägungen analysiert wurden.

Die erste Variable ist die Anzahl der SWS (Ausprägungen: 18 bis 20 SWS vs. 21 SWS und mehr), da eine geringe Anzahl von SWS Studierenden mit zeitlichen Restriktionen eine hohe Flexibilität ermöglicht. Darüber hinaus reduziert eine hohe Anzahl der SWS die zu Verfügung stehende Zeit für das Selbststudium. Im diesem Sinne zeigen auch van den Berg und Hofman (2005, S. 424), dass eine geringere Anzahl von Veranstaltungen pro Semester die Studiendauer reduziert. Der Anteil der Wahlmöglichkeiten gibt den Studierenden die Flexibilität, zeitlich und inhaltlich zwischen mehreren Veranstaltungen zu wählen, und könnte daher die Schwierigkeiten beim Besuch von Lehrveranstaltungen reduzieren (Ausprägungen: 10 Prozent oder weniger Wahlmöglichkeiten vs. über 10 Prozent). Eine regelmäßige Verteilung von Prüfungen über die Semester hinweg kann regelmäßige Lernprozesse unterstützen und Belastungsspitzen vermindern, in denen sich Studierende zwischen individueller Prüfungsvorbereitung und Veranstaltungen entscheiden müssen (Ausprägungen: gleiche Anzahl an Prüfungen pro Semester vs. mehr als zwei Prüfungen Varianz). Ein freier Tag kann schließlich zeitliche Flexibilität schaffen und verlässliche Freiräume für Arbeit oder Lernphasen am Stück schaffen (Ausprägungen: freier Tag vs. kein freier Tag an Wochentagen). Als weiterer Aspekt wurde geprüft, inwieweit die Studiengänge systematisch Präsenzlehre durch virtuelle Lehre ersetzen (BlendedLearning). Die Studiengänge zeigen hierbei jedoch kaum Varianz, sodass Blended-Learning nicht in die Berechnungen aufgenommen werden konnte.

Um die Objektivität zu gewährleisten, führten zwei wissenschaftliche Mitarbeiterinnen des Projektes „Offenes Studienmodell Ludwigshafen“ die Kategorisierung durch. Wenn Abweichungen in den Einschätzungen existierten, wurden diese durch eine dritte Person überprüft. Es wurden nur Studiengänge analysiert, für die mindestens zwölf Studierende im Datensatz vorhanden sind.

\subsection{Auswertungsmethodik}

Das Ziel der Auswertungen war es, Zusammenhänge zwischen den einzelnen Variablen des Modells struktureller Studierbarkeit zu erfassen. Für den Nachweis dieser Zusammenhänge eignen sich neben Mittelwertvergleichen insbesondere multivariate Verfahren, da hierbei der Zusammenhang einer abhängigen Variable mit mehreren unabhängigen Variablen gleichzeitig geprüft werden kann. Die genutzten Verfahren werden im Folgenden dargestellt.

Der Gegenstand der Analysen sind Aspekte der Studierbarkeit, wobei die Bedingungen in den einzelnen Studiengängen die Studierbarkeit stark beeinflussen. Da die Studierenden in Studiengängen gruppiert sind, kommen 
Mehrebenenmodelle zur Anwendung (Sedlmeier und Renkewitz 2013, S. 692). Diese Modelle weisen zwei Ebenen auf, erstens die (Mikro-)Ebene der Studierenden und zweitens die (Makro-)Ebene der Studiengänge. Dabei wird ermittelt, welcher Anteil der Varianz durch die Zugehörigkeit zu einem Studiengang gebunden wird. Im Anschluss prüft das Verfahren außerdem mögliche Unterschiede in der Stärke des Effektes zwischen den Studiengängen (Hox 2010, S. 10 ff.; Langer 2010, S. 732 ff.). Die vorliegenden Daten eignen sich zur Durchführung von Mehrebenenanalysen. Dies hat mehrere Gründe: Erstens sind die Studierenden in 43 Studiengängen eingeschrieben. Damit ist die von Snijders und Bosker (2012, S. 48) formulierte Mindestanzahl an Fällen auf der Makroebene von 20 übertroffen. Zweitens zeigen Signifikanztests, dass die Varianzkomponenten der Konstanten sich unter den Studiengängen unterscheiden und daher Mehrebenenmodelle angemessen sind (Dülmer 2018, S. 28; Snijders und Bosker 2012, 98 ff.). Lediglich in zwei Modellen hat die Konstante keine Varianzkomponente und es wäre eine einfache OLS-Regressionen möglich. Die einzige logistische Regression mit der abhängigen Variable der Studiendauer wird daher nicht als Mehrebenenmodell berechnet. Alle linearen Regressionen werden allerdings als Mehrebenenanalysen durchgeführt, um die Interpretation einheitlich $\mathrm{zu}$ gestalten und der theoretisch begründeten Strukturierung der Daten in Individuen und Studiengänge Rechnung zu tragen. Die Koeffizienten und Signifikanzen werden bei diesem Vorgehen nicht überschätzt.

\section{Interpretationshilfe!}

Die Daten werden mit Mehrebenenmodellen ausgewertet, so dass die Berechnungen die Zugehörigkeit der Studierenden (Mikroebene) in unterschiedlichen Studiengängen (Makroebene) berücksichtigen. Dieses Vorgehen ist notwendig, wenn die befragten Personen sich in verschiedenen Gruppen einteilen lassen - in diesem Fall Studierende nach Studiengängen.

Nach der Entscheidung für Mehrebenenanalysen ist die Analyseart zu spezifizieren. Erstens unterscheiden sich die Varianzkomponenten der Konstanten zwischen den Studiengängen. Daher ist ein Random-Intercept-Modell zu nutzen. Dies bedeutet, dass die Konstanten der Studiengänge im Modell variieren. Zweitens differieren die Effekte der unabhängigen Variablen in ihrer Steigung nicht signifikant, so dass die Steigungskoeffizienten fixiert werden und daher für alle Studierendengruppen gleich sind (Fixed-Slope-Modell). Dieses RandomIntercept-fixed-Slope-Modell wird mit Maximum-Likelihood-Schätzern berechnet. Lediglich in der Mehrebenenregression der abhängigen Variable 
Abbruchneigung (Tabelle 22) wird der Steigungskoeffizient der Variable „Schwierigkeiten der Teilnahme an Prüfungen“ nicht fixiert, da die Steigungskoeffizienten dieser Variable sich signifikant voneinander unter den Studiengängen unterscheiden (Random-Intercept-Random-Slope-Modell). Als deskriptives Maß zur Entscheidung, ob ein Ein- oder Mehrebenenmodell die Daten besser abbildet, wurde in der Vergangenheit der Intraklassen-Korrelation (ICC) zu Rate gezogen. Er prüft, wie groß der Varianzanteil der Makro- bzw. Studiengangsebene an der gesamten Varianz der unabhängigen Variable ausfällt. Da der ICC die die absolute Größe eines Varianzanteils nicht abbildet und im Vergleich zur Signifikanzprüfung der Varianzkomponenten lediglich deskriptiv ist, wird in dieser Arbeit der ICC nicht als Entscheidungsgrundlage für oder gegen Mehrebenenmodelle herangezogen (Dülmer 2018, S. 11). Der ICC wird in den Tabellen aus Gründen der Konvention berichtet.

Die Messung der Varianzaufklärung erfolgt nach Raudenbush und Bryk (2002, S. 85 ff.). Hierbei wird die Varianz auf der Mikro- und Makrobene des Modells mit Regressoren von den Varianzen des Nullmodells ohne Regressoren abgezogen und dieser Wert wiederum von eins subtrahiert. Die Varianzaufklärung kann negative Werte annehmen, wenn das Modell keine „bedeutsamen Variablen auf der Makroebene enthält" (Langer 2010, S. 756). In der Regression der Vereinbarkeitseinschätzung in der Tabelle 18 tritt dieser Fall ein, da das Modell bewusst noch keine Makrovariablen enthält. Die Varianzauklärung kann außerdem hoch sein, wenn die zu erklärende Varianz sehr gering ist (siehe Fernstudiengänge in Tabelle 34).

Bei der Auswahl der Analysemethodik spielt weiterhin das Skalenniveau eine wichtige Rolle. Sind die abhängigen Variablen metrisch oder quasimetrisch skaliert, so kommen lineare Verfahren zur Anwendung. Dies ist aufgrund der 5erLikert-Skalierung bei fast allen abhängigen Variablen in dieser Arbeit der Fall (Carifio und Perla 2007, 114 f.). Die berichteten Koeffizienten sind dann nichtstandardisiert und unter Konstanthaltung der anderen Faktoren als additiv zu interpretieren. Die Vorzeichen weisen auf positive oder negative Zusammenhänge hin.

Die abhängige Variable der geplanten Studiendauer ist nominalskaliert, mit ungleichen Abständen zwischen den Ausprägungen. Diese Ausprägungen werden dichotomisiert mit einer logistischen Regression analysiert, welche die Eintrittswahrscheinlichkeit einer längeren Studiendauer $(\mathrm{RSZ}>1)$ mit der Regelstudienzeit plus einem Semester vergleicht und in Form von Prozentpunkten für den Durchschnitt aller Personen im Datensatz berechnet. Als Koeffizienten dienen durchschnittliche marginale Effekte (AME), die ,einen durchschnittlichen Effekt auf die Wahrscheinlichkeiten an[geben] und [...] nicht von (unkorrelierter) 
unbeobachteter Heterogenität betroffen [sind]“ (Best und Wolf 2010, S. 838). Daher sind AME dazu geeignet, schrittweise aufgebaute Modelle zu vergleichen.

Bei linearen Verfahren wurden die Modellannahmen geprüft: (1) keine Verletzung der Normalverteilungsannahme bzw. Normalverteilung der Residuen, (2) Homoskedastizität, (3) keine Verletzung der Exogenitätsannahme bzw. keine der unabhängigen Variablen korreliert mit dem Fehlerterm, (4) keine Multikollinearität, (5) keine Stichprobenselektivität (Wolf und Best 2010, S. 615 f.). Die Bereiche 3 bis 5 wurden auch für die logistischen Regressionen geprüft. Die Prüfung zeigt, dass die Modellannahmen in der Regel nicht verletzt sind. Eine Ausnahme ist die Normalverteilung der Residuen, die bei der Variable Studienabbruchneigung nicht gegeben ist. In diesem Fall wird dies dementsprechend benannt und methodisch durch robuste Standardfehler behoben. Die Modelle werden schrittweise aufgebaut. Damit kann geprüft werden, ob zusätzliche Variablen eine signifikante Erklärungskraft aufweisen. ${ }^{11}$

Tabelle 16 stellt alle in den Analysen genutzten Variablen vor. Einige der abhängigen Variablen sind in anderen Modellen ebenfalls unabhängige Variablen. Die Ausprägungen der Variablen sind in einigen Fällen nicht identisch mit denen im Fragebogen, da Kategorien zum Zwecke der Vereinfachung zusammengefasst wurden. Die letzten vier Variablen der Tabelle sind Struktur- beziehungsweise Makrovariablen, deren Ausprägungen auf Ebene der Studiengänge festgelegt sind.

${ }^{11}$ Hierfür werden LR-Tests durchgeführt. Ist dieser Test signifikant, ist die Erklärungskraft des unrestringierten Modells mit zusätzlichen Variablen größer als die des restringierten Modells. 
Tabelle 16 Übersicht über die in Regressionen genutzten Variablen. Eigene Darstellung.

\begin{tabular}{|c|c|c|}
\hline Item (abhängige Variablen) & Skala & $\begin{array}{l}\text { Mittelwert (SD) } \\
\text { oder Anteil in \% }\end{array}$ \\
\hline $\begin{array}{l}\text { Meine Verpflichtungen schränken mich bei der } \\
\text { regelmäßigen Teilnahme an Lehrveranstaltungen } \\
\text { ein. }\end{array}$ & \multirow{3}{*}{$\begin{array}{l}\text { 5er-Likert-Skala, } \\
5=\text { trifft voll zu }\end{array}$} & MW: 2,37 (1,14) \\
\hline $\begin{array}{l}\text { Mir fällt es schwer, Zeit zum Lernen neben dem } \\
\text { Studium und meinen anderen Verpflichtungen zu } \\
\text { finden. }\end{array}$ & & MW: 3,17 (1,18) \\
\hline $\begin{array}{l}\text { Trotz meiner Verpflichtungen kann ich } \\
\text { Prüfungen zum geplanten Zeitpunkt ablegen } \\
\text { (rekodiert). }\end{array}$ & & MW: $1,71(0,95)$ \\
\hline $\begin{array}{l}\text { Ich denke ernsthaft darüber nach, mein Studium } \\
\text { ganz aufzugeben. }\end{array}$ & $\begin{array}{l}\text { 5er-Likert-Skala, } \\
5=\text { trifft voll zu }\end{array}$ & MW: $1,38(0,86)$ \\
\hline $\begin{array}{l}\text { Bitte schätzen Sie Ihre Studiendauer. Wie viel } \\
\text { Zeit benötigen Sie voraussichtlich für Ihr } \\
\text { Studium, gemessen an der Regelstudienzeit? }\end{array}$ & $\begin{array}{l}<=\text { Regelstudienzeit }+1 \\
\text { Mehr als } 1 \text { Semester } \\
\text { länger }\end{array}$ & $\begin{array}{l}89 \%(\mathrm{~N}=1.009) \\
11 \%(\mathrm{~N}=121)\end{array}$ \\
\hline $\begin{array}{l}\text { Skala Zufriedenheit mit Studienbedingungen } \\
\text { Skala Zufriedenheit mit Studienbelastungen }\end{array}$ & $\begin{array}{l}\text { 5er-Likert-Skala, } \\
5=\text { hohe Zufriedenheit }\end{array}$ & $\begin{array}{l}\text { MW: 2,88 }(0,97) \\
\text { MW: 3,48 }(0,93)\end{array}$ \\
\hline $\begin{array}{l}\text { Wenn Sie erneut ein Studium beginnen würden, } \\
\text { welche Art der Studienorganisation würden Sie } \\
\text { wählen? } \\
\text { - Vollzeitstudium } \\
\text { - Teilzeitstudium } \\
\text { - Wechsel Voll- und Teilzeit } \\
\text { - Fernstudium Vollzeit } \\
\text { - Fernstudium Teilzeit } \\
\text { - Duales Studium }\end{array}$ & $\begin{array}{l}\text { 5er-Likert-Skala, } \\
5=\text { sicher ja }\end{array}$ & $\begin{array}{l}M W=4,46(0,89) \\
M W=2,67(1,25) \\
M W=2,84(1,43) \\
M W=1,73(1,38) \\
M W=1,67(1,04) \\
M W=3,44(1,04)\end{array}$ \\
\hline Item (unabhängige Variablen) & Skala & $\begin{array}{l}\text { Mittelwert (SD) } \\
\text { oder Anteil in \% }\end{array}$ \\
\hline Was ist Ihr Geschlecht? & $\begin{array}{l}\text { Männlich } \\
\text { Weiblich }\end{array}$ & $\begin{array}{l}38,5 \%(\mathrm{~N}=452) \\
61,5 \%(\mathrm{~N}=723)\end{array}$ \\
\hline Wie alt sind Sie? & Alter & MW $=25$ Jahre \\
\hline $\begin{array}{l}\text { Haben Ihre Eltern ein Hochschulstudium } \\
\text { abgeschlossen? }\end{array}$ & $\begin{array}{l}\text { Nein } \\
\mathrm{Ja}\end{array}$ & $\begin{array}{l}65,1 \%(\mathrm{~N}=768) \\
34,9 \%(\mathrm{~N}=412) \\
\end{array}$ \\
\hline $\begin{array}{l}\text { Sind Sie gesundheitlich beeinträchtigt, und hat } \\
\text { diese Beeinträchtigung Auswirkungen auf Ihr } \\
\text { Studium? Mehrfachnennungen möglich }\end{array}$ & $\begin{array}{l}\text { Nein oder } \\
\text { Beeinträchtigung ohne } \\
\text { Studieneinschränkung } \\
\text { Beeinträchtigung mit } \\
\text { Studieneinschränkung }\end{array}$ & $\begin{array}{l}94,3 \% \\
(\mathrm{~N}=1.181) \\
5,7 \%(\mathrm{~N}=71)\end{array}$ \\
\hline $\begin{array}{l}\text { In welchem Umfang sind Sie derzeit in der } \\
\text { Vorlesungszeit erwerbstätig? }\end{array}$ & $\begin{array}{l}0 \text { Stunden } \\
1-10 \text { Stunden } \\
11-20 \text { Stunden } \\
\text { Mehr als } 20 \text { Stunden } \\
\end{array}$ & $\begin{array}{l}45,2 \%(\mathrm{~N}=565) \\
23,5 \%(\mathrm{~N}=294) \\
25,5 \%(\mathrm{~N}=320) \\
05,8 \%(\mathrm{~N}=73)\end{array}$ \\
\hline $\begin{array}{l}\text { Haben Sie Kinder? } \\
\text { Wer ist hauptsächlich für die Betreuung Ihrer } \\
\text { Kinder zuständig? }\end{array}$ & $\begin{array}{l}\text { Kind unter } 16 \text { Jahren } \\
\text { Betreue selbst } \\
\text { Betreue nicht selbst }\end{array}$ & $\begin{array}{l}1,1 \%(\mathrm{~N}=13) \\
2,8 \%(\mathrm{~N}=36)\end{array}$ \\
\hline
\end{tabular}


Pflegen Sie nahe Angehörige, die aus Altersoder Krankheitsgründen dauerhaft pflegebedürftig sind?

Pflege...

... ohne zeitliche

$2,0 \%(\mathrm{~N}=25)$

Einschränkung im

Studium

$2,1 \%(\mathrm{~N}=26)$

... mit zeitlicher

Einschränkung im

Studium

\begin{tabular}{|c|c|c|}
\hline Index zeitliche Restriktion & $\begin{array}{l}\text { Keine } \\
\text { Geringe } \\
\text { Mittlere } \\
\text { Hohe }\end{array}$ & $\begin{array}{l}39,9 \%(\mathrm{~N}=500) \\
23,4 \%(\mathrm{~N}=293) \\
25,5 \%(\mathrm{~N}=319) \\
11,2 \%(\mathrm{~N}=140) \\
\end{array}$ \\
\hline $\begin{array}{l}\text { Hochschulzugangsberechtigung: Bitte kreuzen } \\
\text { Sie alle allgemeinbildenden/beruflichen } \\
\text { Abschlüsse an. }\end{array}$ & $\begin{array}{l}\text { Abitur } \\
\text { Fachhochschulreife } \\
\text { Beruflich qualifiziert } \\
\text { Ausländisch }\end{array}$ & $\begin{array}{l}59,5 \%(\mathrm{~N}=715) \\
29,7 \%(\mathrm{~N}=356) \\
06,2 \%(\mathrm{~N}=75) \\
04,6 \%(\mathrm{~N}=55)\end{array}$ \\
\hline $\begin{array}{l}\text { Ich fühle mich durch das Studium oft müde und } \\
\text { abgespannt. }\end{array}$ & $\begin{array}{l}\text { 5er-Likert-Skala, } \\
5=\text { trifft voll zu }\end{array}$ & MW: $3,03(0,96)$ \\
\hline $\begin{array}{l}\text { Skala soziale Interaktion } \\
\text { Skala akademische Interaktion }\end{array}$ & $\begin{array}{l}\text { 5er-Likert-Skala, } \\
5=\text { hohe Interaktion }\end{array}$ & $\begin{array}{l}\text { MW: 3,69(0,75) } \\
\text { MW: } 2,84(0,69)\end{array}$ \\
\hline Skala Vereinbarkeit & $\begin{array}{l}\text { 5er-Likert-Skala, } \\
5=\text { gute Vereinbarkeit }\end{array}$ & MW: $2,74(0,99)$ \\
\hline $\begin{array}{l}\text { Wie bewerten Sie den... } \\
\text {...Umfang der Lehrveranstaltungen (SWS)? } \\
\text {...Anteil der Wahlmöglichkeiten? }\end{array}$ & 5er-Skala, $5=$ zu hoch & $\begin{array}{l}\text { MW. 3,25 }(0,66) \\
\text { MW: } 1,86(0,78)\end{array}$ \\
\hline Durchschnittliche Anzahl der SWS* & $\begin{array}{l}18-20 \text { SWS } \\
\text { Über } 20 \text { SWS }\end{array}$ & $\begin{array}{l}\mathrm{N}=312(\mathrm{~N}=9) \\
\mathrm{N}=860(\mathrm{~N}=20)\end{array}$ \\
\hline Anteil Wahlmöglichkeiten im Studiengang* & $\begin{array}{l}\text { Mehr als 10 Prozent } \\
<10 \% \text { Wahl }\end{array}$ & $\begin{array}{l}25,7 \%(\mathrm{~N}=6) \\
74,3 \%(\mathrm{~N}=19)\end{array}$ \\
\hline Freie Tage im Stundenplan (WiSe 2015/16)* & $\begin{array}{l}1 \text { freier Tag an } \\
\text { Wochentagen Kein freier } \\
\text { Tag }\end{array}$ & $\begin{array}{l}\mathrm{N}=179(\mathrm{~N}=4) \\
\mathrm{N}=767(\mathrm{~N}=15)\end{array}$ \\
\hline $\begin{array}{l}\text { Verteilung der Prüfungen im Studiengang } \\
\text { zwischen den Semestern* }\end{array}$ & $\begin{array}{l}\text { Varianz an Prüfungen } \\
\text { Maximal eine } \\
\text { Zwei Prüfungen oder } \\
\text { mehr }\end{array}$ & $\begin{array}{l}42,6 \%(\mathrm{~N}=14) \\
57,4 \%(\mathrm{~N}=11)\end{array}$ \\
\hline
\end{tabular}


Open Access Dieses Kapitel wird unter der Creative Commons Namensnennung 4.0 International Lizenz (http://creativecommons.org/licenses/by/4.0/deed.de) veröffentlicht, welche die Nutzung, Vervielfältigung, Bearbeitung, Verbreitung und Wiedergabe in jeglichem Medium und Format erlaubt, sofern Sie den/die ursprünglichen Autor(en) und die Quelle ordnungsgemäß nennen, einen Link zur Creative Commons Lizenz beifügen und angeben, ob Änderungen vorgenommen wurden.

Die in diesem Kapitel enthaltenen Bilder und sonstiges Drittmaterial unterliegen ebenfalls der genannten Creative Commons Lizenz, sofern sich aus der Abbildungslegende nichts anderes ergibt. Sofern das betreffende Material nicht unter der genannten Creative Commons Lizenz steht und die betreffende Handlung nicht nach gesetzlichen Vorschriften erlaubt ist, ist für die oben aufgeführten Weiterverwendungen des Materials die Einwilligung des jeweiligen Rechteinhabers einzuholen.

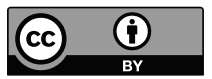

